\title{
Quantum error avoiding codes verses quantum error correcting codes
}

\author{
Lu-Ming Duan*and Guang-Can Guo ${ }^{\dagger}$
}

\begin{abstract}
A general theory of quantum error avoiding codes is established, and new light is shed on the relation between quantum error avoiding and correcting codes. Quantum error avoiding codes are found to be a special type of highly degenerate quantum error correcting codes. A combination of the ideas of correcting and avoiding quantum errors may yield better codes. We give a practical example.
\end{abstract}

PACS numbers: $03.75,89.70 .+\mathrm{c}, 03.65 . \mathrm{Bz}$

\footnotetext{
*Electronic address: lmduan@ustc.edu.cn

${ }^{\dagger}$ Electronic address: gcguo@sunlx06.nsc.ustc.edu.cn
} 
In quantum computation or communication, it is essentially important to maintain coherence of a quantum system [1]. In reality, however, decoherence due to the interaction of the system with noisy environment is inevitable [2]. It is discovered that the quantum redundant coding is the most efficient way to combat decoherence. Until now, many kinds of quantum codes has been devised [3-24]. According to their principles, quantum codes can be divided into three classes, i.e., quantum error correcting codes (QECCs) [3-15], quantum error preventing codes (QEPCs) [16,17], and quantum error avoiding codes (QEACs) [18-23]. QECCs are capable of detecting and correcting quantum errors. QEPCs just detect errors. From the quantum Zeno effect, quadratic noise can be suppressed by frequent error detections $[16,17]$. QEACs avoid quantum errors by encoding input states into coherence-preserving states. These schemes do not need to detect and correct errors. They are useful with specific noise models $[19,20]$.

A general theory of QECCs has been established in Ref. [8]. In this paper, we develop a general theory of QEACs. Necessary and sufficient conditions for QEACs are obtained. We find an interesting connection between QEACs and QECCs. QEACs can be regarded as a special type of highly degenerate QECCs, and furthermore, if degeneracy of a QECC attains the maximum, the code necessarily becomes a QEAC. The existing QECCs of practical importance all belong to the class of non-degenerate QECCs [3-15]. These codes are devised to correct error after occurrence of the error. They do not avoid errors. In contrast, the QEACs avoid errors, but have no ability to correct errors. A combination of the ideas of correcting and avoiding errors may yield better codes. We give examples by devising a class of quantum codes in a practical circumstance. These codes, which are found to be more efficient than the QEACs and the existing 
non-degenerate QECCs, provide interesting examples of degenerate QECCs.

We start by developing a general formalism for QEACs. A quantum information system generally consists of many qubits. The system inevitably interacts with noisy environment. The total Hamiltonian describing the interacting system is denoted by $H_{t o t}$, which may include free evolution of the qubits, qubit-qubit interactions, and qubit-environment coupling. $H_{t o t}$ can be divided into two parts, i.e.,

$$
H_{t o t}=H_{c}+H_{u c}
$$

The first part $H_{c}$ refers to the Hamiltonian that is controllable, such as the free evolution of the qubits and quantum logic operations. The second part $H_{u c}$ represents the uncontrollable Hamiltonian, such as some noisy interactions between the qubits and the coupling of the qubits to noisy environment. In reality, the Hamiltonian $H_{c}$ makes the system evolve in a controllable way and results in quantum computation. The Hamiltonian $H_{u c}$ results in noise and decoherence, which should be eliminated if we want to bring quantum computation into practice.

We discuss the noisy interaction $H_{u c}$ in the interaction picture. The interaction Hamiltonian has the form (setting $\hbar=1)$

$$
H_{I}(t)=e^{-i H_{c} t} H_{u c} e^{i H_{c} t} .
$$

Under this Hamiltonian, after a certain time the reduced density operator of the system evolves from $\rho_{i}$ to $\rho_{f}=\hat{S}\left(\rho_{i}\right)$, where $\hat{S}$ is the superoperator associated with the noisy interaction. In the case where the environment is not initially entangled with the system, $\rho_{f}$ can be written in the form [24]

$$
\rho_{f}=\sum_{a} A_{a} \rho_{i} A_{a}^{+}
$$


where the linear operators $A_{a}$ satisfy the condition

$$
\sum_{a} A_{a}^{+} A_{a}=I
$$

and $I$ is the unit operator. All the $A_{a}$ are called interaction operators. For a given evolution $S$, the choice of the operator family $\left\{A_{a}\right\}$ is not unique. We choose the smallest family by requiring that the operators $A_{a}$ are linearly independent in the whole Hilbert space of the qubits. Under this requirement, the number of elements in the family $\left\{A_{a}\right\}$ is uniquely defined, but concrete forms of the $A_{a}$ are not determined yet. Look at the following transformation:

$$
B_{b}=\sum_{a} x_{b a} A_{a}
$$

where the coefficients $x_{b a}$ satisfy the condition $\sum_{b} x_{b a}^{*} x_{b a^{\prime}}=\delta_{a a^{\prime}}$, i.e., the matrix $X=\left[x_{b a}\right]$ is unitary. Under the transformation (5), it is evident that the operator family $\left\{B_{b}\right\}$ also satisfies $\rho_{f}=\sum_{b} B_{b} \rho_{i} B_{b}^{+}$and $\sum_{b} B_{b}^{+} B_{b}=I$. Hence, $\left\{B_{b}\right\}$ is also a realization of the evolution $S$. In the operator-sum representation (3), all the operator families linked by the unitary transformation (5) are equivalent. Because of this equivalence, for a given evolution $S$ we can choose the simplest operator family. In the following, without loss of generality, we choose that $A_{0}=\gamma_{0} I$ with $0<\gamma_{0}<1$. This choice is possible if $\rho_{i}$ and $\rho_{f}$ are not orthogonal to each other. The interaction operators which are not proportional to $I$ are called error operators.

To give an accurate definition of QEACs, it is convenient to use the notion of fidelities. For a pure input state $\rho_{i}=\left|\Psi_{i}\right\rangle\left\langle\Psi_{i}\right|$, which are subjected to noise described by the operator family $\left\{A_{a}\right\}$, the input-output state fidelity is defined by

$$
F\left(\left|\Psi_{i}\right\rangle,\left\{A_{a}\right\}\right)=\left\langle\Psi_{i}\left|\rho_{f}\right| \Psi_{i}\right\rangle=\sum_{a}\left|\left\langle\Psi_{i}\left|A_{a}\right| \Psi_{i}\right\rangle\right|^{2}
$$


A code $C$ is defined as a subspace of the whole Hilbert space $H$ of the qubits. The code fidelity is measured by

$$
F\left(C,\left\{A_{a}\right\}\right)=\min _{|\Psi\rangle \in C} F\left(|\Psi\rangle,\left\{A_{a}\right\}\right)
$$

The code $C$ is defined to be a QEAC if $C$ is a maximal linear subspace of $H$ which has the property that the code fidelity

$$
F\left(C,\left\{A_{a}\right\}\right)=\min _{|\Psi\rangle \in C} \sum_{a}\left|\left\langle\Psi\left|A_{a}\right| \Psi\right\rangle\right|^{2}=1
$$

Suppose that $M$ and $N$ are the dimensions of the code $C$ and of the whole Hilbert space $H$, respectively. The efficiency of the code is given by $\eta=\frac{\log _{2} M}{\log _{2} N}$.

The QEAC is characterized by the following theorem:

Theorem 1. The code $C$ can be extended to a QEAC iff (if and only if) $C$ is a co-eigenspace of all the interaction operators $A_{a}$.

This condition is more general than the ones given in Refs. [21] and [23], which are sufficient but not necessary. Theorem1 gives a necessary and sufficient condition for QEACs.

Proof. Assume that $C$ can be extended to a QEAC. From the definition (8), for an arbitrary state $|\Psi\rangle \in C$, we have

$$
\sum_{a}\left|\left\langle\Psi\left|A_{a}\right| \Psi\right\rangle\right|^{2}=1
$$

The state $A_{a}|\Psi\rangle$ can always be decomposed as

$$
A_{a}|\Psi\rangle=\gamma_{a}|\Psi\rangle+\gamma_{a}^{\perp}\left|\Psi^{\perp}\right\rangle
$$

where $\gamma_{a}, \gamma_{a}^{\perp}$ are coefficients, and $\left|\Psi^{\perp}\right\rangle$ denotes a normalized state orthogonal to the state $|\Psi\rangle$. Equations (9) and (10) yield

$$
\sum_{a}\left|\gamma_{a}\right|^{2}=1
$$


On the other hand, from Eqs. (4) and (10), it follows that

$$
\sum_{a}\left(\left|\gamma_{a}\right|^{2}+\left|\gamma_{a}^{\perp}\right|^{2}\right)=1
$$

Hence, we have $\gamma_{a}^{\perp}=0$, i.e., $|\Psi\rangle$ is a co-eigenstate of all the interaction operators $A_{a}$, with the eigenvalues $\gamma_{a}$, respectively. The eigenvalues $\gamma_{a}$ should be independent of the state $|\Psi\rangle$. If $\left|\Psi_{1}\right\rangle \in C$ and $\left|\Psi_{2}\right\rangle \in C$, from the linearity of $C, c_{1}\left|\Psi_{1}\right\rangle+c_{2}\left|\Psi_{2}\right\rangle$ also belongs to $C$. So, $\left|\Psi_{1}\right\rangle,\left|\Psi_{2}\right\rangle$ and $c_{1}\left|\Psi_{1}\right\rangle+c_{2}\left|\Psi_{2}\right\rangle$ are co-eigenstates of the operators $A_{a}$. This is possible iff they have the same eigenvalues; thus $C$ is a co-eigenspace of all the interaction operators.

The converse of the theorem is straightforward. If $C$ is a co-eigenspace of all the interaction operators, with the eigenvalues denoted by $\gamma_{a}$, respectively, for an arbitrary state $|\Psi\rangle \in C$, obviously we have

$$
\sum_{a}\left|\left\langle\Psi\left|A_{a}\right| \Psi\right\rangle\right|^{2}=\sum_{a}\left|\gamma_{a}\right|^{2}=\sum_{a}\left\langle\Psi\left|A_{a}^{+} A_{a}\right| \Psi\right\rangle=1
$$

Hence $F\left(C,\left\{A_{a}\right\}\right)=1$, and $C$ can be extended to a QEAC. This completes the proof of the theorem.

It is interesting to compare QEACs with QECCs. Unlike QEACs, QECCs are influenced by the error operators. But the influence can be eliminated and the encoded state can be perfectly recovered by applying an appropriate recovery operator. From the definition, we see that QEACs can be regarded as a special type of QECCs, which do not need any recovery operations. Hence, a QEAC should also satisfy the condition for QECCs. The necessary and sufficient condition for QECCs has been given in [8]. Assume that $C^{\prime}$ is a code, and $\left\{A_{a}\right\}$ denotes the family of interaction operators. The code $C^{\prime}$ can be extended to a QECC iff for all basisvectors $\left|i_{L}\right\rangle,\left|j_{L}\right\rangle$ of $C^{\prime}$ and operators $A_{a}, A_{b}$ in $\left\{A_{a}\right\}$

$$
\left\langle i_{L}\left|A_{a}^{\dagger} A_{b}\right| j_{L}\right\rangle=\gamma_{a b} \delta_{i j}
$$


where the coefficients $\gamma_{a b}$ should be independent of the basisvectors. The coefficient matrix $\Gamma=\left[\gamma_{a b}\right]$ is obviously Hermitian, but its form is not uniquely defined, since the choice of the interaction operators is not unique. All the operator families linked by the unitary transformation (5) are equivalent. Under these transformations, the Hermitian coefficient matrix $\Gamma$ can always be cast into a diagonal matrix, with the eigenvalues being positive real numbers. If all the eigenvalues of $\Gamma$ do not equal zero, the code is called a non-degenerate QECC. In contrast, if some eigenvalues equal zero, or equivalently, if some lines of the coefficient matrix $\Gamma$ are linearly dependent, the code is degenerate. All the discovered QECCs devised in practical circumstances belong to the class of non-degenerate codes. A formal example of degenerate QECCs was given in [8].

Obviously, QEACs should also satisfy the condition (14); but not all the codes satisfying Eq. (14) are QEACs. What kind of restrictions need be added for QEACs? The additional restriction is shown by the following theorem, which provides another form of the necessary and sufficient condition for QEACs.

Theorem 2. The code $C$ can be extended to a QEAC iff for all basisvectors $\left|i_{L}\right\rangle,\left|j_{L}\right\rangle$ of $C$ and interaction operators $A_{a}, A_{b}$ in the family $\left\{A_{a}\right\}$

$$
\left\langle i_{L}\left|A_{a}^{\dagger} A_{b}\right| j_{L}\right\rangle=\gamma_{a}^{*} \gamma_{b} \delta_{i j}
$$

Proof. Assume that $C$ can be extended to a QEAC. From theorem 1, for an arbitrary $\left|i_{L}\right\rangle \in C$ and $A_{a} \in\left\{A_{a}\right\}$, we have

$$
A_{a}\left|i_{L}\right\rangle=\gamma_{a}\left|i_{L}\right\rangle
$$

thus Eq. (15) holds. Conversely, if Eq. (15) holds, Eq. (4) yields

$$
\sum_{a}\left|\gamma_{a}\right|^{2}=1
$$


Suppose that $|\Psi\rangle$ is an arbitrary state in the subspace $C$. From Eq. (15), it follows that

$$
\left\langle\Psi\left|A_{a}^{\dagger} A_{b}\right| \Psi\right\rangle=\gamma_{a}^{*} \gamma_{b}
$$

The choice of the operator family $\left\{A_{a}\right\}$ is not unique, and we can always choose that $A_{0}=\gamma_{0} I$. Let $A_{b}$ in Eq. (17) equal $A_{0}$, then we have

$$
\left\langle\Psi\left|A_{a}\right| \Psi\right\rangle=\gamma_{a}
$$

The code fidelity

$$
F\left(C,\left\{A_{a}\right\}\right)=\min _{|\Psi\rangle \in C} \sum_{a}\left|\left\langle\Psi\left|A_{a}\right| \Psi\right\rangle\right|^{2}=\sum_{a}\left|\gamma_{a}\right|^{2}=1
$$

thus $C$ can be extended to a QEAC. This completes the proof.

Theorem 2 has an interesting corollary. We know that QEACs can be regarded as special QECCs. It is natural to ask in what circumstances QECCs reduce to QEACs. This question is answered by the following corollary of theorem 2 .

Corollary. The QECC $C$ reduces to a QEAC iff the coefficients $\gamma_{a b}$ in Eq. (14) can be decomposed as $\gamma_{a}^{*} \gamma_{b}$.

If $\gamma_{a b}$ is decomposed as $\gamma_{a}^{*} \gamma_{b}$, the rank of the coefficient matrix $\Gamma=\left[\gamma_{a b}\right]$ is not larger than one, and at most one eigenvalue of the matrix $\Gamma$ does not equal zero. Hence, in this circumstance the code is highly degenerate. We therefore have the following conclusion: If degeneracy of a QECC attains the minimum, the code is a non-degenerate QECC; Conversely, if the degeneracy attains the maximum, the code becomes a QEAC.

QEACs and non-degenerate QECCs are two extremes. Are there intermediate circumstances? In the following, we consider a practical decoherence model. For this model, the optimal code is neither a QEAC nor a non-degenerate QECC, but a combination of them. The code correct and avoid errors at the same time, and 
it provides an interesting example for degenerate QECCs of practical importance. (To our knowledge, this is the first practical example).

All the discovered QEACs assume the collective decoherence model $[19,20]$. Suppose we have $L$ qubits. In the collective decoherence model, the error operators are described by $A^{+}=\gamma^{+} \sum_{l=1}^{L} \sigma_{l}^{+}, A^{-}=\gamma^{-} \sum_{l=1}^{L} \sigma_{l}^{-}$, and $A^{z}=\gamma^{z} \sum_{l=1}^{L} \sigma_{l}^{z}$, where $\vec{\sigma}_{l}$ are Pauli's operators. The three operators $A^{+}, A^{-}$, and $A^{z}$, together with $A_{0}=\gamma_{0} I$ make a complete family of the interaction operators. To avoid all the collective errors, four is the least number of qubits to encode one qubit of information [20]. In the circumstance of collective decoherence, QEACs are more efficient than the non-degenerate QECCs. The latter needs at least five qubits to encode a bit of quantum information [6].

Collective decoherence results form the assumption that the distance between the qubits is very small so that it is less than the effective wave length of the noise field $[18,19]$. It is most possible for the closely spaced adjacent qubits to satisfy this assumption. Hence, here we assume that every two adjacent qubits (called a qubit-pair) are decohered collectively; but the qubits in different qubit-pairs are allowed to decohere in an arbitrary manner, possibly independently, possibly cooperatively. Suppose that we have $2 L$ qubits, denoted by $1,1^{\prime}, 2,2^{\prime}, \cdots$, and $L, L^{\prime}$, respectively. The $l$ and $l^{\prime}(l=1,2, \cdots, L)$ qubits are decohered collectively. In our decoherence model, the error operators are described by

$$
A_{l}^{\alpha}=\gamma_{l}^{\alpha}\left(\sigma_{l}^{\alpha}+\sigma_{l^{\prime}}^{\alpha}\right)
$$

with $l=1,2, \cdots, L$ and $\alpha= \pm$, or $z$. For this decoherence model, the nondegenerate QECCs need at least five qubits to encode one qubit of information; and it is impossible to devise any QEACs, for the co-eigenspace of the error operators $A_{l}^{\alpha}$ is of only one dimension with the sole basisvector $\left|\Psi_{i}\right\rangle=$ 
$\otimes_{l}\left[\frac{1}{\sqrt{2}}\left(|01\rangle_{l l^{\prime}}-|10\rangle_{l l^{\prime}}\right)\right]$, where $|0\rangle_{l}$ and $|1\rangle_{l}$ are two eigenstates of the operator $\sigma_{l}^{z}$. However, a combination of the ideas of correcting and avoiding errors can yield better codes. In fact, four qubits are enough to encode one qubit of information. Suppose that the first qubit 1 is in an arbitrary input state $\left|\Psi_{i}\right\rangle_{1}=c_{0}|0\rangle_{1}+c_{1}|1\rangle_{1}$. The ancillary qubits $1^{\prime}, 2$, and $2^{\prime}$ are prepared in the states $|1\rangle_{1^{\prime}},|0\rangle_{2}$, and $|1\rangle_{2^{\prime}}$, respectively. The encoding is given by the following operation :

$$
\left.\left|\Psi_{i}\right\rangle_{1} \otimes|101\rangle_{1^{\prime} 22^{\prime}} \stackrel{C_{11^{\prime}} C_{12} C_{12^{\prime}}}{\longrightarrow} \mid \Psi \text { enc }\right\rangle=c_{0}|0101\rangle_{11^{\prime} 22^{\prime}}+c_{1}|1010\rangle_{11^{\prime} 22^{\prime}}
$$

where all the $C_{i j}$ represent the controlled-NOT (CNOT) operation, with the first subscript of $C_{i j}$ referring to the control bit and the second to the target. After this encoding, obviously we have $A_{1}^{z} \mid \Psi$ enc $\rangle=A_{2}^{z} \mid \Psi$ enc $\rangle=0$, where $A_{l}^{z}(l=1,2)$ are defined by Eq. (20). Hence, the errors $A_{1}^{z}$ and $A_{2}^{z}$ are avoided. The remaining errors $A_{1}^{ \pm}$and $A_{2}^{ \pm}$can be easily detected and corrected. We make a quantum nondemolition measurement of the operators $\sigma_{l}^{z}+\sigma_{l^{\prime}}^{z}$. If the measurement outcome is \pm 2 for an $l$, the error $A_{l}^{ \pm}$takes place; and it is readily corrected by performing some quantum CNOT operations. For this decoherence model, the four-bit code (21) can be easily proven to be optimal by showing that three bits are not enough to encode one qubit of information.

The above code can be extended straightforwardly to multi-qubit circumstances. The general input state of $L$ qubits is expressed as

$$
\left|\Psi_{L}\right\rangle=\sum_{\left\{i_{l}\right\}} c_{\left\{i_{l}\right\}}\left|\left\{i_{l}\right\}\right\rangle
$$

where $\left\{i_{l}\right\}$ denotes $i_{1}, i_{2}, \cdots, i_{L}$, and $\left|\left\{i_{l}\right\}\right\rangle$ represents $\left|i_{1}\right\rangle \otimes\left|i_{2}\right\rangle \otimes \cdots \otimes\left|i_{L}\right\rangle$ with $i_{l}=0$ or 1 . We use $2 L+2$ qubits to encode $L$ qubits of information. The state (22) is encoded into the following state of $L+1$ qubit-pairs

$$
\left|\Psi_{2 L+2}\right\rangle_{\mathrm{enc}}=\sum_{\left\{i_{l}\right\}} c_{\left\{i_{l}\right\}}\left|\left\{i_{l}, \bar{i}_{l^{\prime}}\right\}\right\rangle \otimes\left|i_{L+1}, \bar{i}_{L^{\prime}+1}\right\rangle
$$


where $\left|\left\{i_{l}, \bar{i}_{l^{\prime}}\right\}\right\rangle$ denotes $\left|i_{1}\right\rangle \otimes\left|\bar{i}_{1^{\prime}}\right\rangle \otimes\left|i_{2}\right\rangle \otimes\left|\bar{i}_{2^{\prime}}\right\rangle \otimes \cdots \otimes\left|i_{L}\right\rangle \otimes\left|\bar{i}_{L^{\prime}}\right\rangle$ with $\bar{i}_{l^{\prime}}=1-i_{l}$.

The state $\left|i_{L+1}, \bar{i}_{L^{\prime}+1}\right\rangle$ of the $(L+1)$ th qubit-pair should satisfy $\sum_{l=1}^{L+1} i_{l}=0 \mathrm{mod}$ 2. Through the encoding (23), all the errors $A_{l}^{z}$ are avoided. The remaining errors $A_{l}^{ \pm}$can be detected and corrected by the procedure very similar to that in the single qubit circumstance. For a large $L$, the efficiency of the code is approximately $\frac{1}{2}$. In the case of $L \geq 2$, the code (23) is not necessarily optimal; but it has the advantage of being very simple, and easy to encode, decode, and to detect the error syndrome.

All the discovered QEACs assume the collective decoherence model. Before ending the paper, we emphasize that QEACs may also find their application in other decoherence models. For example, it is possible to avoid some correlated errors by QEACs. As a simple example, we assume that there are only two qubits, subject to the following correlated errors. The error operators are given by $A_{1}=\sigma_{1}^{+} \sigma_{2}^{-}$, and $A_{2}=\sigma_{2}^{+} \sigma_{1}^{-}$. These errors can be avoided by the encoding

$$
\begin{gathered}
|0\rangle \rightarrow|00\rangle, \\
|1\rangle \rightarrow|11\rangle .
\end{gathered}
$$

One qubit of information is encoded. This simple example suggests that QEACs may have wide use. It is an interesting question to find further applications of QEACs in other practical decoherence models.

\section{Acknowledgment}

This project was supported by the National Nature Science Foundation of China. 


\section{References}

[1] D. P. DiVincenzo, Science 270, 255 (1995).

[2] W. G. Unruh, Phys. Rev. A 51, 992 (1995).

[3] P. W. Shor, Phys. Rev. A 52, R2493 (1995).

[4] A. M. Steane, Phys. Rev. Lett. 77, 793 (1996); Proc. R. Soc. London A 452, 2551 (1996).

[5] A. R. Calderbank and P. W. Shor, Phys. Rev. A 54, 1098 (1996).

[6] R. Laflamme, C. Miguel, J. P. Paz, and W. H. Zurek, Phys. Rev. Lett. 77, 198 (1996).

[7] C. H. Bennett, D. P. DiVincenzo, J. A. Smolin, and W. K. Wootters, Phys. Rev. A 54, 3824 (1996).

[8] E. Knill and R. Laflamme, Phys. Rev. A 55, 900 (1997).

[9] D. Gottesman, Phys. Rev. A 54, 1844 (1996).

[10] A. R. Calderbank, E. M. Rains, P. W. Shor. and N. J. A. Sloane, Phys. Rev. Lett. 78, 465 (1997).

[11] D. P. DiVincenzo and P. W. Shor, Phys. Rev. Lett. 77, 3260 (1996).

[12] A. M. Steane, Phys. Rev. Lett. 78, 2252 (1997).

[13] H. F. Chau, Phys. Rev. A 56, R1 (1997).

[14] S. Lloyd and J. -J. E. Slotine, Phys. Rev. Lett. 80, 4088 (1998). 
[15] S. L. Braunstein, Phys. Rev. Lett. 80, 4084 (1998).

[16] L. Vaidman, L. Goldenberg, S. Wiesner, Phys. Rev A 54, R1745 (1996).

[17] L. M. Duan and G. C. Guo, Phys. Rev. A 57, 2399 (1998).

[18] G. M. Palma, K. A. Suominen, and A. K. Ekert, Proc. R. Soc. London A 452,567 (1996).

[19] L. M. Duan and G. C. Guo, Phys. Rev. Lett. 79, 1953 (1997); Phys. Rev. A 57, 737 (1998).

[20] P. Zanardi and M. Rasetti, Phys. Rev. Lett 79, 3306 (1997).

[21] P. Zanardi and M. Rasetti, Mod. Phys. Lett. B 11, 1085 (1997).

[22] L. M. Duan and G. C. Guo, LANL eprint quant-ph/9804014, to appear in Phys. Rev. A 58 (4), (1998).

[23] D. A. Lidar, I. L. Chuang, and K. B. Whaley, LANL eprint quant$\mathrm{ph} / 9807004$.

[24] K. Kraus, States, Effects, and Options (Springer-Verlag, Berlin ,1983). 